\title{
Exposure of a Dengue Vector to Tea and Its Waste: Survival, Developmental Consequences, and Significance for Pest Management
}

ABSTRACT: Dengue mosquitoes are evolving into a broader global public health menace, with relentless outbreaks and the rise in number of Zika virus disease cases as reminders of the continued hazard associated with Aedes vectors. The use of chemical insecticides-the principal strategy against mosquito vectors-has been greatly impeded due to the development of insecticide resistance and the shrinking spectrum of effective agents. Therefore, there is a pressing need for new chemistries for vector control. Tea contains hundreds of chemicals, and its waste, which has become a growing global environmental problem, is almost as rich in toxicants as green leaves. This paper presents the toxic and sublethal effects of different crude extracts of tea on Aedes albopictus. The survival rates of larvae exposed to tea extracts, especially fresh tea extract (FTE), were markedly lower than those in the control treatment group. In addition to this immediate toxicity against different developmental stages, the extracts tested caused a broad range of sublethal effects. The developmental time was clearly longer in containers with tea, especially in those with young larvae (YL) and FTE. Among the survivors, pupation success was reduced in containers with tea, which also produced low adult emergence rates with increasing tea concentration. The production of eggs tended to be reduced in females derived from the tea treatment groups. These indirect effects of tea extracts on Ae. albopictus exhibited different patterns according to the exposed larval stage. Taken together, these findings indicate that tea and its waste affect most key components of Ae. albopictus vectorial capacity and may be useful for dengue control. Reusing tea waste in vector control could also be a practical solution to the problems associated with its pollution.

KEYWORDS: dengue, tea, mosquito, Ae. albopictus

\section{INTRODUCTION}

Dengue infects 50-100 million people worldwide, resulting in thousands of deaths annually. ${ }^{1}$ This disease is regarded as a continuous threat to public health and has potential for much greater impact in the future with 5-6 billion people predicted to be at risk by 2085 . $^{2}$ Previously considered a less important dengue vector than Aedes aegypti, Aedes albopictus has been the vector responsible for several outbreaks worldwide. ${ }^{3}$

Efforts to control these vectors have mainly relied on the use of chemical insecticides, but the development of insecticide resistance to the main families of insecticides used for vector control worldwide has hampered the success of these programs. ${ }^{4}$ Due to the lack of effective licensed vaccines and specific medications, ${ }^{5}$ further studies to identify novel agents with mosquitocidal properties are needed. Despite substantial efforts to manage insecticide resistance by developing new products, there are a number of difficulties related to cost and time for research, and very few insecticides are available that are simultaneously economical, effective, and nonhazardous to humans. ${ }^{6}$ Therefore, it is an important strategy to target highly available and chemically rich materials.

Tea (Camellia sinensis) is one of the most popular beverages in the world. ${ }^{7}$ This popularity is accompanied by the disposal of huge amounts of tea waste into the environment. ${ }^{8}$ For example, $1,650,000$ cups of tea are consumed every day in the United Kingdom, which results in the production of 370,000 tonnes of tea bag waste every year. ${ }^{9}$ In India, every $100 \mathrm{~kg}$ of green tea leaves processed produces $4 \mathrm{~kg}$ of refuse. ${ }^{10}$ Tea leaves contain over 700 chemicals, $^{11}$ many of which have detrimental effects on insects. These agents include flavonoids, which have been reported to exert increased anti-cytochrome P450-dependent ecdysone 20-monooxygenase activities in drosophilids, lepidopterans, and mosquitoes; ${ }^{12}$ phenolic compounds such as chlorogenic acid, which reduces the amino acid bioaccessibility and decreases digestion in various insects; ${ }^{13}$ alkaloids that deter feeding and kill insects; ${ }^{14}$ methylxanthines that cause death; ${ }^{15}$ and caffeine, which inhibits beetle feeding and kills certain insects. ${ }^{16}$ Here, we examined whether tea leachates can alter larval development and its outcomes as well as some reproductive fitness traits of Ae. albopictus.

\section{EXPERIMENTAL DETAILS}

Ae. albopictus mosquitoes utilized in this study originated from a colony kept under conditions of controlled relative humidity $(75 \pm$ $5 \%)$, photoperiod [13:10 h (light/dark) with $1 \mathrm{~h}$ of dusk], and temperature $\left(29 \pm 3.0^{\circ} \mathrm{C}\right)$ in the insectary of the School of Biological Sciences, University Sains Malaysia. To obtain experimental immature stages, samples of eggs from the stock were immersed in dechlorinated water. Samples of 100 newly hatched larvae (NHL) fed $1 \mathrm{~mL}$ of the food suspension described above and that had been allowed to develop for 2 days were used as young larvae (YL). Samples of 100 NHL allowed to grow for $4-5$ days and fed $3 \mathrm{~mL}$ of larval food were regarded as old larvae (OL). NHL that were fed every 2 days with increasing amounts of food suspension $(1 \mathrm{~mL}$ of food suspension for NHL, $2 \mathrm{~mL} 2$ days later, $3 \mathrm{~mL}$ for the next food provision) that reached the pupal stage (PUP) were used as experimental subjects.

The tea used in this study was Ever Green G401/G402 (Shanghai Tiantan International Trading Co., Ltd., China). Several studies have been carried out using remnants from a first extraction to mimic food waste and to assess the insecticidal properties of their aqueous extracts. ${ }^{17-20}$ Therefore, here, we utilized used tea to mimic tea waste. The different test tea extracts were prepared according to a modification of the method reported previously for cigarette butt ${ }^{12}$ and coffee ${ }^{17}$ extracts. Briefly, $4 \mathrm{~g}$ of fresh green tea leaves was dipped in

Received: April 5, 2016

Accepted: April 26, 2016 